\title{
Efecto del tratamiento térmico en harina de avena utilizada en la sustitución de harina de trigo para la elaboración de pan
}

\author{
Heat treatment effect on oat flour used in the substitution of wheat flour for bread- making
}

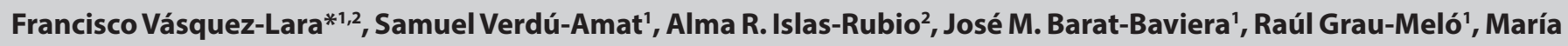
del Carmen Granados-Nevárez², Benjamín Ramírez Wong ${ }^{3}$

Departamento de Tecnología de Alimentos. Universidad Politécnica de Valencia. Camino de Vera s/n, C.P. 46022, Valencia, España.

2 Coordinación de Tecnología de Alimentos de Origen Vegetal. Centro de Investigación en Alimentación y Desarrollo, A.C. Carretera Gustavo Enrique Astiazarán Rosas No. 46, Col. La Victoria, C.P. 83304, Hermosillo, Sonora, México.

3 Departamento de Investigación y Posgrado en Alimentos. Universidad de Sonora, CP 83000, Hermosillo, Sonora, México.
\end{abstract}

\section{RESUMEN}

La harina de avena fue tratada térmicamente a 80 , 100 y $130{ }^{\circ} \mathrm{C}$ durante 30 min para después ser utilizada en sustitución del 10 y $20 \%$ de harina de trigo para la elaboración de pan. Se determinó capacidad de retención de agua, perfil de viscosidad, volumen específico y análisis de perfil de textura a los 0, 2 y 7 días de elaborado el pan. Los parámetros de viscosidad fueron afectados por los dos niveles de sustitución de harina de avena y el tratamiento térmico. Al utilizar harina de avena tratada a $100{ }^{\circ} \mathrm{C}$ a 10 y $20 \%$ de sustitución, el volumen específico de pan aumentó (2.64 y $2.75 \mathrm{~cm}^{3} / \mathrm{g}$ ). El perfil de textura, también se vio afectado por el nivel de sustitución y el tratamiento térmico a los 7 días de almacenamiento, la firmeza del pan disminuyó cuando se usó $10 \%$ de harina de avena tratada a $130^{\circ} \mathrm{C}(12.86 \mathrm{~N})$ en comparación al elaborado con harina de trigo (19.63 N). Los tratamientos térmicos en harina de avena empleada para sustituir la harina de trigo pueden ser una alternativa importante para mejorar la calidad en productos de panificación.

Palabras clave: Harina de trigo, harina de avena, tratamiento térmico, sustitución.

\section{ABSTRACT}

Oat flour was heat treated at 80,100 or $130{ }^{\circ} \mathrm{C}$ for $30 \mathrm{~min}$, then used to replace 10 or $20 \%$ wheat flour in bread-making process. Water retention capacity, viscosity profile, specific volume, and texture profile analysis were determined at 0, 2 and 7 days after bread-making. Viscosity parameters were affected by the two levels of oat flour substitution and heat treatment. When using oat flour treated at $100{ }^{\circ} \mathrm{C}$ and substitution level of 10 and $20 \%$, the specific volume of bread increased ( 2.64 and $2.75 \mathrm{~cm}^{3} / \mathrm{g}$ ). The texture profile was also affected by the level of substitution and heat treatment at 7 days of storage, the firmness of the bread decreased when $10 \%$ of oat flour treated at $130{ }^{\circ} \mathrm{C}(12.86 \mathrm{~N})$ was used in comparison to that made with wheat flour (19.63 $\mathrm{N})$. Heat treatments in oat flour used to replace wheat flour can be an important alternative to improve the quality of bread-making products.

*Autor para correspondencia: Francisco Vsásquez-Lara

Correo electrónico: fvas@ciad.mx

Recibido: 19 de octubre de 2020

Aceptado: 15 de enero de 2021
Key words: Wheat flour, oat flour, heat treatment, substitution.

\section{INTRODUCCIÓN}

Después de la molienda, la harina de trigo normalmente recibe ciertos tratamientos para mejorar algunas de sus propiedades. En particular puede ser la adición de aditivos como agentes de maduración u oxidantes (bromato de potasio, ácido ascórbico, azodicarbonamida o sulfato cúprico), blanqueamiento (peróxido o gas de cloro) o ingredientes para promover la fermentación. Los agentes de maduración causan cambios fisicoquímicos en la harina, propiedades similares a las realizadas en el proceso de envejecimiento natural que afectan las propiedades reológicas de la masa y, por lo tanto, la capacidad de retención de gas del gluten (SernaSaldívar, 1996). Los agentes blanqueadores que reducen el contenido de pigmentos amarillos causan una coloración blanca en las harinas.

Un complemento al tratamiento anterior puede ser la aplicación de tratamientos térmicos en harinas que han sido utilizados ampliamente en la industria harinera mucho antes de la eliminación del proceso de cloración (blanqueo de harina con dióxido de cloro) a principios de los 90s (Chesterton et al., 2015). La harina tratada con calor tiene muchas aplicaciones dentro del procesamiento de alimentos, entre ellas: la preparación de alimentos para bebés, salsas, sopas, cremas y rellenos, espesante, alimentos instantáneos, alimentos precocinados, confitería, mayonesa, pan y productos derivados de trigo, entre otros. Russo \& Doe (1970), fueron los primeros en patentar este proceso usando un rango de temperatura de 100 a $115^{\circ} \mathrm{C}$ durante $60 \mathrm{~min}$. Otros investigadores (Cauvain et al., 1976) sugirieron un tratamiento térmico en el grano de trigo y sémola hasta un contenido de humedad del $6 \%$. Hanamoto \& Bean (1979), patentaron el método para producir harinas tratadas térmicamente, utilizando una temperatura de $71{ }^{\circ} \mathrm{C}$ por 4 días. Estudios de Nakamura et al. (2008) observaron un aumento en el volumen del pastel Kasutera (pastel popular en Japón) cuando la harina de trigo se trató a $120{ }^{\circ} \mathrm{C}$ durante $30 \mathrm{~min}$. Otras harinas de cereales 
que han sido expuestas al tratamiento térmico son la harina de sorgo. Estudios de Marston et al. (2016) utilizaron harina de sorgo para preparar pan sin gluten, que se trató con calor a $95^{\circ} \mathrm{C}$ y $125^{\circ} \mathrm{C}$ durante 15,30 o $45 \mathrm{~min}$, observando efectos importantes en la viscosidad de la masa, volumen específico del pan y en la miga. También se realizaron tratamientos térmicos en almidones. Jiranuntakul et al. (2011), aplicaron temperaturas de $100{ }^{\circ} \mathrm{C}$ por $16 \mathrm{~h}$ a diferentes almidones de arroz, papa, maíz, entre otros, observando cambios significativos en la temperatura de gelatinización y la viscosidad. El tratamiento térmico en harinas y la eliminación de la humedad pueden modificar las propiedades físicas, reológicas y biológicas de las harinas. La eliminación de la humedad es esencial para lograr los cambios necesarios, sin embargo, es importante ajustar de nuevo la humedad de la harina para lograr resultados óptimos de cocción (Neill et al., 2012). El tratamiento térmico afecta la extensibilidad de la masa y la absorción de agua, además de la gelatinización de almidón. En las harinas utilizadas en la fabricación de pan se ha observado que el tratamiento térmico ha aumentado la resistencia, la viscosidad y la rigidez (Gelinas et al., 2001). Estos efectos tienden a aumentar la elasticidad de la masa y producir un efecto positivo en el volumen del pan (Pyler, 1988). En base a lo anterior, el objetivo de este trabajo de investigación, fue evaluar el efecto en las características texturales, reológicas y calidad del pan que tiene el uso de harina de avena tratada térmicamente en la sustitución de harina de trigo.

\section{MATERIAL Y MÉTODOS Materia prima}

La harina de avena $(\mathrm{HA})$ comercial (marca Biográ) se obtuvo de un productor local, La Carabasseta, Valencia, España. Su composición fue de: $11.3 \%$ de proteína, $8.0 \%$ de grasa, $12.6 \%$ de humedad y $0.92 \%$ de cenizas (base seca). La harina de trigo comercial (HT) fue suministrada por un productor local, Molí de Picó-Harinas Segura S. L. Valencia, España. Su composición fue: $12.7 \%$ de proteína, $1.0 \%$ de grasa, $13.09 \%$ de humedad y $0.32 \%$ de cenizas (base seca). Para mantener la homogeneidad en el tamaño de partícula, la HA se molió en un molino de acero inoxidable (Retsch GmbH, ZM 200, Haan, Alemania), hasta que se logró la misma distribución del tamaño de partícula que la HT utilizada (malla \#80). Las harinas compuestas se obtuvieron sustituyendo 10 y $20 \%$ $(p / p)$ de HT con HA. El resto de los ingredientes utilizados para panificación fueron: aceite de girasol (Koipesol Semillas, S.L, España), levadura prensada (Saccharomyces cerevisiae, Lesafre Ibérica, S.A, España), azúcar blanco ( $\geq 99.8 \%$ de sacarosa, Azucarera Ebro, S.L España) y sal (sal marina refinada $\geq$ $97 \% \mathrm{NaCl}$ Salinera Española S.A, España), que se compraron en tiendas locales.

\section{Tratamientos térmicos en harina de avena}

La HA fue sometida a tratamientos térmicos de 80 , 100 y $130^{\circ} \mathrm{C}$ durante $30 \mathrm{~min}$ en una estufa con aire caliente de convección forzada (Neill et al., 2012). La temperatura se controló con termómetro digital (rango 0-300 드, resolución $0.1^{\circ} \mathrm{C}$, precisión $\pm 0.2^{\circ} \mathrm{C}$ ). Durante este proceso, la humedad se redujo de $15 \%$ al $10.5 \%$. La pérdida de humedad se calculó gravimétricamente en función de la diferencia de masa entre los pesos, antes y después del tratamiento térmico. Para recuperar los valores de humedad originales, las harinas se colocaron en una cámara (KBF720 Binder Tuttlingen Alemania) con humedad y temperatura controladas (15\% HR y $25^{\circ} \mathrm{C}$ ) por $48 \mathrm{~h}$.

\section{Capacidad de retención de agua (CRA)}

Se realizó de acuerdo con el método 56-11.02 (AACC, 2000). Las muestras de harina ( $5 \mathrm{~g}$ ) se pesaron en tubos de centrífuga $(50 \mathrm{~mL})$ con tapones de rosca. Luego se añadieron $25.0 \mathrm{~mL}$ de agua y se agitó vigorosamente $5 \mathrm{~s}$ para suspender la harina. Las muestras se mantuvieron durante 20 min en un mezclador vórtex con agitación intermitente a los 5, 10, 15 y 20 min, seguido de 15 min de centrifugación 1000 x g (Centrifuge Beckman Coulter Allegra X-30R, Brea, California, EE. UU.). El sobrenadante se desechó y el sedimento húmedo obtenido se dejó decantar por 10 min, posteriormente se pesó. La CRA se calculó según lo descrito por Haynes et al. (2009).

\section{Perfil de viscosidad (Rapid Visco Analyser, RVA)}

El perfil de viscosidad de las harinas se obtuvo por el método 76-21.02 (AACC, 2000). Se utilizó el viscosímetro RVA-S4 (Rapid Visco Analyzer Super 4, Newport Scientific, Warriewood, Australia). Las mezclas de harinas se analizaron sobre la base de $3.5 \mathrm{~g} \pm 0.01 \mathrm{~g}$ de muestra ajustada al $14 \%$ de humedad. La cantidad de agua incorporada fue de $25 \mathrm{~g}$ $\pm 0.01 \mathrm{~g}$. El perfil de prueba fue de una temperatura inicial de $50{ }^{\circ} \mathrm{C}$ y 960 RPM, y disminuyó a 160 RPM a los 10 s. En el min 1 de la prueba, la temperatura fue de $50{ }^{\circ} \mathrm{C}$, después se aumentó a $95^{\circ} \mathrm{C}$ durante 4.42 min y permaneció así hasta los $7.42 \mathrm{~min}$. Al min 11, la temperatura bajó a $50{ }^{\circ} \mathrm{C}$ y se mantuvo durante 2 min más hasta que la prueba finalizó a los 13 min. Los parámetros obtenidos fueron: temperatura de gelatinización, viscosidad pico, mínima, de rompimiento, de restitución y final.

\section{Proceso de panificación}

La formulación utilizada para preparar la masa de pan fue la siguiente: $56 \%$ de harina (HT o mezclas de harinas), 2 $\%$ de aceite de girasol refinado, $2 \%$ de levadura prensada comercial, $4 \%$ de azúcar blanca, $1.5 \%$ de sal y $34.5 \%$ de agua (Verdú et al., 2015). El proceso se llevó a cabo mezclando todos los ingredientes en el mezclador de alimentos Thermomix TM31 (Vorwerk, Alemania). Los componentes líquidos (agua y aceite), azúcar y sal se mezclaron 2 min a 37 ${ }^{\circ} \mathrm{C}$. Se añadió levadura para mezclar a la misma temperatura por 30 s. Se agregó harina y se mezcló con los ingredientes de acuerdo con un programa predeterminado para hacer una masa homogénea para panificación. El sistema se basa en mezclar los ingredientes con giros aleatorios de la hélice del mezclador en ambas direcciones (550 RPM). Este proceso se aplicó por $4.5 \mathrm{~min}$ a $37^{\circ} \mathrm{C}$. Después se colocaron $250 \mathrm{~g}$ de 
masa en el molde de metal $(8 \times 8 \times 30 \mathrm{~cm})$ para llevar a cabo la fermentación. Esta se realizó en una cámara con humedad y temperatura controlada (KBF720, Binder, Tuttlingen, Alemania). Las condiciones del proceso de fermentación fueron 40 ${ }^{\circ} \mathrm{C}$ y $90 \%$ de HR. Las masas fueron fermentadas durante $1 \mathrm{~h}$. El proceso de horneado se realizó al finalizar la fermentación. Los moldes conteniendo la masa se colocaron en el horno (De Longhi, Italia) pre-calentado a $200^{\circ} \mathrm{C}$. El tiempo de horneado fue de $15 \mathrm{~min}$.

\section{Evaluaciones en pan}

Se midió el volumen de pan mediante el método de desplazamiento de semillas de colza (Cordero et al., 2020). Lo anterior permitió determinar el volumen específico $\left(\mathrm{cm}^{3} / \mathrm{g}\right)$ que se calculó en base a la relación entre el volumen $\left(\mathrm{cm}^{3}\right)$ y el peso de pan (g). El proceso de horneado también se estudió en función de la pérdida de peso producida (g). Se cuantificó por la diferencia entre el peso de la masa (g) y el peso de pan (g).

\section{Actividad de agua $\left(A_{w}\right)$}

La actividad de agua $\left(A_{w}\right)$ de las migas se determinó con el higrómetro Aqualab ${ }^{\circledR}$ de punto de rocío (DECAGÓN Aqualab CX-2, Pullman, WA, EE. UU.).

\section{Análisis del perfil de textura (TPA)}

El análisis del perfil de textura (TPA) se realizó a los 0 , 2 y 7 días de almacenamiento a temperatura ambiente, siguiendo el método utilizado por Miñarro et al. (2012), donde se obtuvieron tres rebanadas de la sección transversal de $12.5 \mathrm{~mm}$ de espesor del centro de cada pan. El análisis de perfil de textura se realizó en el analizador de textura TA-TX2 (Stable Micro Systems, Surrey, Reino Unido). Se usó una celda de carga de $25 \mathrm{~kg}$ y la velocidad del ensayo fue de $1.7 \mathrm{~mm} / \mathrm{s}$ para comprimir el centro de la miga del pan $50 \%$ de su altura. El tiempo entre compresiones fue de $5 \mathrm{~s}$.

\section{Análisis estadístico}

Los resultados se analizaron aplicando un análisis de varianza (ANOVA) de una vía utilizando el procedimiento de comparación múltiple para determinar que medias son significativamente diferentes de otras. Para discriminar entre las medias se utilizó el procedimiento de diferencia mínima significativa (LSD) de Fisher. Para el análisis se usó el programa Statgraphics Centurion XVI con un nivel de confianza del $95 \%$.

\section{RESULTADOS Y DISCUSIÓN \\ Capacidad de retención de agua (CRA)}

En harinas de trigo esta propiedad es utilizada para determinar su calidad y capacidad para formar una masa visco-elástica, la cual es fundamental en la industria de los alimentos ya que puede determinar sus propiedades funcionales. La Figura 1A, muestra los resultados en la CRA cuando HT se sustituyó con 10 y $20 \%$ de HA tratada térmicamente a 80,100 y $130^{\circ} \mathrm{C}$. Se observó una tendencia a disminuir la
CRA al incrementar el tratamiento térmico en HA y el nivel de sustitución de HT. Todos los valores obtenidos resultaron menores al que se obtuvo en HT (69.03\%). Esto posiblemente ocasionado por un pre-cocimiento del almidón a causa de los tratamientos térmicos a la que fue sometida la HA utilizada en los diferentes niveles de sustitución.

También se ha observado que al aplicar altas temperaturas en harinas es posible llevar a cabo una desnaturalización de proteínas que son afectadas en la capacidad de retener agua (Protonotariou et al., 2014). Otros estudios realizados por Berton et al. (2002) encontraron que la capacidad de retención de agua depende principalmente de la cantidad de almidón dañado, cuando utilizaron harina de trigo en sus investigaciones. Estudios realizados por Duyvejonck et al. (2011) también encontraron una relación muy importante entre el nivel de daño en el almidón y la capacidad de retención de agua en harinas de trigos europeos. Estudios realizados por Protonotariou et al. (2014), observaron un efecto altamente significativo entre la distribución de tamaño de partículas y propiedades funcionales en harinas de trigo como lo es la capacidad de retención de agua. Pauly et al. (2013) concluyeron que cuanto mayor es el área de superficie específica por unidad de peso, mayor es la tasa de hidratación y absorción de agua en harinas. Por lo anterior, es que la CRA juega un papel muy importante en las propiedades de los alimentos como puede ser la textura del producto final.

\section{Perfil de viscosidad (Rapid Visco Analyser, RVA)}

El RVA normalmente se relaciona con el comportamiento del almidón, sin embargo, existen otros componentes muy importantes que pueden influir en esta prueba como son las proteínas y lípidos. Estos componentes interactúan con el almidón y afectan la medición de viscosidad en el RVA (Batey, 2009). En este trabajo se estableció el perfil de viscosidad de HT, además de las mezclas de harina de $\mathrm{HT}$ sustituidas con $\mathrm{HA}$ tratada térmicamente a 80,100 y $130^{\circ} \mathrm{C}$ a niveles de 10 y $20 \%$.

La Figura 1B, muestra el efecto que tuvo la sustitución de HT con HA tratada térmicamente sobre la temperatura de gelatinización $\left({ }^{\circ} \mathrm{C}\right)$. Se observó una reducción significativa en HT $\left(68.95^{\circ} \mathrm{C}\right)$, con respecto a la que se obtuvo cuando HA se usó en la sustitución de $\mathrm{HT}$ al 10 y $20 \%$, tratada a 80 y $100^{\circ} \mathrm{C}$. Sin embargo, al aumentar el tratamiento térmico a $130^{\circ} \mathrm{C}$, para los dos niveles de sustitución este parámetro se redujo considerablemente $\left(67.67\right.$ y $\left.69.35^{\circ} \mathrm{C}\right)$. Este comportamiento puede explicarse por la gelatinización previa de almidón cuando se emplean altas temperaturas, en este caso $130^{\circ} \mathrm{C}$, generando así una disminución en el contenido de gránulos de almidón disponibles para gelatinizar. El hinchamiento del gránulo de almidón comienza tan pronto como el gránulo empieza a hidratarse. La hidratación es un precursor esencial para la gelatinización. En la mayoría de los almidones, la gelatinización completa es un requisito previo esencial para que el cocimiento (empaste) sea observado (Batey, 2009). Estudios realizados por Neill et al. (2012) observaron éste mismo comportamiento al utilizar tratamientos térmicos en $\mathrm{HT}$. 
La Figura 1C, muestra los efectos que tuvieron los tratamientos térmicos mencionados anteriormente en la viscosidad pico de las harinas sustituidas con HA. La viscosidad pico, es la viscosidad máxima registrada por una muestra durante el calentamiento. Con respecto a este parámetro se observó un aumento significativo al utilizar HA al 10 y $20 \%$ tratada a 80 y $100{ }^{\circ} \mathrm{C}$, con respecto a HT (2245 CP). Al aumentar el tratamiento a $130^{\circ} \mathrm{C}$, la disminución en viscosidad fue más evidente cuando la sustitución fue del $20 \%$ (2217 cP), incluso llegando a ser inferior a la obtenida para HT. Estudios realizados por Neill et al. (2012) en HT tratada térmicamente muestran disminuciones de $320 \mathrm{BU}$ a $220 \mathrm{BU}$ empleando el Viscógrafo Brabender. Ozawa et al. (2009) estudiaron el efecto del tratamiento térmico $\left(120^{\circ} \mathrm{C}\right.$ durante $30,60,90$ y 120 min) sobre el almidón de HT observando una disminución en la viscosidad pico de 694 a 646 RVU.

Los gránulos que tienen una alta capacidad de hinchamiento también tienden a tener una alta viscosidad. La velocidad de calentamiento tiene un efecto importante sobre la viscosidad pico. Contrario a lo anterior, la reducción en el hinchamiento de los gránulos de almidón es indicativo de las bajas viscosidades pico y la disminución en la ruptura de los gránulos de almidón (Ktenioudaki et al., 2013).

Otro parámetro muy importante que se analizó fue la viscosidad final que es la formada por el gel durante el enfriamiento sucediendo una re-asociación entre moléculas de almidón, principalmente amilosa que resulta en la formación de un gel que incrementa dicha viscosidad (Lei et al., 2008). La Figura 1D, muestra los resultados relacionados con la viscosidad final de HT y de sus mezclas con HA. Se observaron valores muy similares cuando la HT se reemplazó por 10 y $20 \%$ de HA tratada a 80 y $100{ }^{\circ} \mathrm{C}$, respectivamente. Al aumentar la temperatura del tratamiento térmico en HA a 130
${ }^{\circ} \mathrm{C}$, en los dos niveles de sustitución se puede observar que la viscosidad disminuye, siendo muy parecida a la registrada por HT (2660 cP). Este comportamiento puede generarse por una pérdida en la organización granular del almidón, lo que resulta en una disminución de la gelatinización, en este caso en particular como resultado de la aplicación de tratamientos con alto calor. Además de la gelatinización previa del almidón, las proteínas del gluten sufren pérdida de propiedades funcionales debido a su desnaturalización (Prakash \& Haridas, 1999).

Otros resultados relacionados con el perfil de viscosidad de HT y de las mezclas de harinas son mostrados en la Tabla 1. Los valores de viscosidad mínima mostraron diferencias significativas entre los obtenidos en las sustituciones y HT (1421 cP). Se observaron también aumentos en este parámetro de viscosidad cuando HT se sustituyó con 10 y $20 \%$ de HA tratada a 80 y $100^{\circ} \mathrm{C}$, y cuando se aumentó la temperatura del tratamiento a $130^{\circ} \mathrm{C}$ se apreció una tendencia a disminuir, incluso alcanzando valores menores a los de HT. Lo anterior se observó en ambos niveles de sustitución. Con relación a la viscosidad de rompimiento, esta mostró una ligera tendencia a incrementar a medida que aumentó el tratamiento térmico al que fue sometida HA utilizada en la sustitución de HT. Se observó también que al utilizar HA al 20 \% tratada a 80 y 100 ${ }^{\circ} \mathrm{C}$ no mostraron diferencias significativas con respecto a HT (823 cP). En lo que respecta a la viscosidad de restitución, se observaron diferencias significativas cuando HT (1238 cP) fue sustituida por HA tratada térmicamente a 80, 100 y 130 ${ }^{\circ} \mathrm{C}$, en los dos niveles de sustitución. Para este parámetro de viscosidad, el comportamiento fue muy similar al observado en la viscosidad de restitución, donde las sustituciones de 10 y $20 \%$ con HA tratada a 80 y $100{ }^{\circ} \mathrm{C}$ no mostraron diferencias significativas entre ellas.

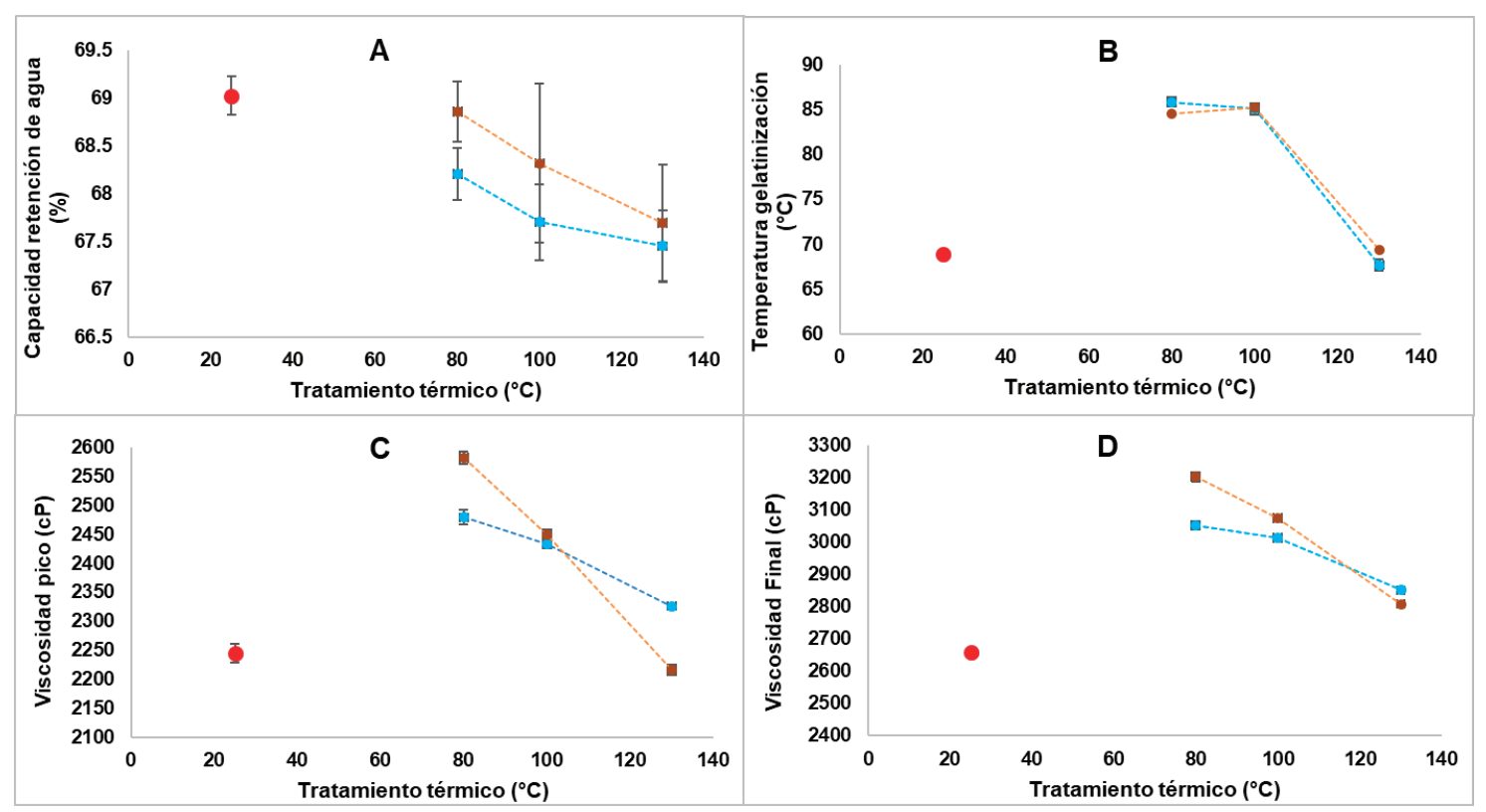

Figura 1. Capacidad de retención de agua (A), temperatura de gelatinización (B), viscosidad pico (C) y viscosidad final (D) de $H T(\bullet)$ y de $\mathrm{HT}$ sustituida al $10 \%(\bullet)$ y $20 \%(\bullet)$ con $\mathrm{HA}$ tratada térmicamente a 80,100 y $130^{\circ} \mathrm{C}$.

Figure 1. Water retention capacity (A), gelatinization temperature (B), peak viscosity (C) and final viscosity (D) of $\mathrm{HT}(\bullet)$ and HT substituted at $10 \%(\bullet)$ and $20 \%(\bullet)$ with heat treated $\mathrm{HA}\left(80,100\right.$ and $\left.130^{\circ} \mathrm{C}\right)$. 
Tabla 1. Viscosidad mínima, rompimiento y restitución en HT y HT sustituida al 10 y $20 \%$ con HA tratada térmicamente a 80,100 y $130^{\circ} \mathrm{C}$.

Table 1. Results of minimum viscosity, breakdown and restitution in $\mathrm{HT}$, and $\mathrm{HT}$ substituted at 10 and $20 \%$ with heat treated $\mathrm{HA}\left(80,100\right.$ and $\left.130^{\circ} \mathrm{C}\right)$.

\begin{tabular}{lccccc}
\hline & & Tratamiento & \multicolumn{3}{c}{$\begin{array}{c}\text { Viscosidad } \\
\text { (cP) }\end{array}$} \\
\cline { 4 - 6 } Harina & Sustitución (\%) & Térmico $\left({ }^{\circ} \mathrm{C}\right)$ & Mínima & Rompimiento & Restitución \\
\hline Trigo & & & $1421 \pm 4.9 \mathrm{c}$ & $823 \pm 10.6 \mathrm{a}$ & $1238 \pm 10.6 \mathrm{a}$ \\
\hline Avena & 10 & 80 & $1633 \pm 0.7 \mathrm{e}$ & $846 \pm 7.1 \mathrm{c}$ & $1414 \pm 11.3 \mathrm{~b}$ \\
\hline Avena & 10 & 100 & $1610 \pm 4.2 \mathrm{~d}$ & $849 \pm 3.5 \mathrm{c}$ & $1408 \pm 0 \mathrm{~b}$ \\
\hline Avena & 10 & 130 & $1393 \pm 4.9 \mathrm{~b}$ & $933 \pm 5.6 \mathrm{~d}$ & $1453 \pm 8.4 \mathrm{~d}$ \\
& & & & & \\
\hline Avena & 20 & 80 & $1781 \pm 4.2 \mathrm{f}$ & $821 \pm 9.8 \mathrm{a}$ & $1437 \pm 4.9 \mathrm{~cd}$ \\
\hline Avena & 20 & 100 & $1623 \pm 4.9 \mathrm{e}$ & $825 \pm 3.5 \mathrm{ab}$ & $1452 \pm 4.2 \mathrm{~d}$ \\
\hline Avena & 20 & 130 & $1378 \pm 9.1 \mathrm{a}$ & $843 \pm 9.8 \mathrm{bc}$ & $1434 \pm 5.6 \mathrm{c}$ \\
\hline
\end{tabular}

$\mathrm{CP}$, centipoise. Letra diferente dentro de la misma columna expresan diferencia significativa $p$ $<0.05$.

\section{Evaluaciones en pan}

Los resultados de las mediciones de volumen específico y pérdida de peso de panes obtenidos de la sustitución de $\mathrm{HT}$ con HA tratada térmicamente a 80,100 y $130^{\circ} \mathrm{C}$ durante 30 min a niveles de sustitución de 10 y $20 \%$ son mostrados en la Tabla 2.

Se observó el aumento en el volumen específico cuando se usó la sustitución de 10 y $20 \%$ de HA tratada a 80,100 y $130^{\circ} \mathrm{C}$ con respecto al obtenido de $\mathrm{HT}\left(2.22 \mathrm{~cm}^{3} / \mathrm{g}\right)$, sin embargo, estos incrementos fueron mayores cuando se utilizó la sustitución del $20 \%$ de $\mathrm{HA}$ tratada a $100^{\circ} \mathrm{C}$. El tratamiento térmico de la $\mathrm{HA}$ a $130^{\circ} \mathrm{C}$ mostró una ligera disminución en el volumen específico para los dos niveles de sustitución. Sin embargo, todavía son mayores a los obtenidos en HT. Estudios realizados por Nakamura et al. (2008) indicaron un aumento gradual en el volumen del pastel kasutera (pastel de esponja japonesa) al tratar la harina térmicamente a $120^{\circ} \mathrm{C}$ durante 30 min. Neill et al. (2012) también se observaron aumentos en la altura del pastel cuando la harina se trató a 120 y $130^{\circ} \mathrm{C}$ durante $30 \mathrm{~min}$. Los parámetros de calidad como el volumen y la textura pueden atribuirse a las propiedades reológicas de la masa. Estas propiedades pueden verse afectadas por la naturaleza hidrofóbica en el almidón primario que ocurre durante el tratamiento térmico de la harina (Nakamura et al., 2008). Los estudios sobre harina de trigo han demostrado que el tratamiento térmico puede aumentar la viscosidad de la masa y mejorar las propiedades de horneado de la harina (Catterall, 2001). Lo anterior puede ser originado a partir de la desnaturalización por calor de las proteínas en la superficie del gránulo de almidón, exponiendo más cadenas laterales hidrofóbas de aminoácidos que están ocultas entre las proteínas nativas (Catterall, 2001).

Los resultados de la pérdida de peso mostraron aumentos significativos cuando la sustitución de HT se utilizó el 10 y $20 \%$ de $\mathrm{HA}$ tratada térmicamente a $80^{\circ} \mathrm{C}$. A medida que aumentó el tratamiento en $\mathrm{HA}$, se observó una disminución en la pérdida de peso, alcanzando los valores más bajos cuando se trató a $130{ }^{\circ} \mathrm{C}$ en los dos niveles de sustitución, incluso más bajo que el observado en HT (13.3 \%). Este comportamiento puede explicarse en estudios realizados

Tabla 2. Volumen específico $\left(\mathrm{cm}^{3} / \mathrm{g}\right)$, pérdida de peso (\%) y actividad de agua $\left(A_{w}\right)$ en HT y HT sustituida al 10 y $20 \%$ con HA tratada térmicamente a 80,100 y $130^{\circ} \mathrm{C}$.

Table 2. Results of specific volume $\left(\mathrm{cm}^{3} / \mathrm{g}\right)$, weight loss $(\%)$ and water activity $\left(A_{w}\right)$ in HT and HT substituted at 10 and $20 \%$ with heat treated $\mathrm{HA}\left(80,100\right.$ and $\left.130^{\circ} \mathrm{C}\right)$.

\begin{tabular}{lccccc}
\hline Harina & Sustitución (\%) & $\begin{array}{c}\text { Tratamiento } \\
\text { Térmico }\left({ }^{\circ} \mathbf{C}\right)\end{array}$ & $\begin{array}{c}\text { Volumen } \\
\text { Específico }\left(\mathbf{c m}^{3} / \mathbf{g}\right)\end{array}$ & $\begin{array}{c}\text { Pérdida de } \\
\text { Peso }(\%)\end{array}$ & $\begin{array}{c}\text { Actividad de } \\
\text { Agua }\left(\mathbf{A}_{\mathrm{w}}\right)\end{array}$ \\
\hline Trigo & & & $2.22 \pm 0.04 \mathrm{a}$ & $13.3 \pm 0.02 \mathrm{~b}$ & $0.958 \pm 0.00 \mathrm{bc}$ \\
\hline Avena & 10 & 80 & $2.45 \pm 0.05 \mathrm{C}$ & $13.8 \pm 0.12 \mathrm{~cd}$ & $0.958 \pm 0.01 \mathrm{bc}$ \\
\hline Avena & 10 & 100 & $2.64 \pm 0.03 \mathrm{e}$ & $13.5 \pm 0.22 \mathrm{bc}$ & $0.959 \pm 0.01 \mathrm{~cd}$ \\
\hline Avena & 10 & 130 & $2.36 \pm 0.02 \mathrm{~b}$ & $12.7 \pm 0.18 \mathrm{a}$ & $0.962 \pm 0.00 \mathrm{~d}$ \\
\hline Avena & 20 & 80 & $2.55 \pm 0.03 \mathrm{~d}$ & $14.1 \pm 0.09 \mathrm{~d}$ & $0.953 \pm 0.01 \mathrm{a}$ \\
\hline Avena & 20 & 100 & $2.76 \pm 0.04 \mathrm{f}$ & $13.6 \pm 0.10 \mathrm{bc}$ & $0.952 \pm 0.01 \mathrm{a}$ \\
\hline Avena & 20 & 130 & $2.48 \pm 0.03 \mathrm{C}$ & $12.7 \pm 0.43 \mathrm{a}$ & $0.955 \pm 0.01 \mathrm{ab}$ \\
\hline
\end{tabular}

Los resultados mostrados son el promedio de 5 repeticiones \pm desviación estándard. Letra diferente dentro de la misma columna expresan diferencia significativa $p<0.05$. 
por Neill et al. (2012) donde observaron que el tratamiento térmico redujo la extensibilidad del gluten y generó una mayor retención de humedad. Estudios realizados por Verdú et al. (2015) en harina de trigo para elaborar pan sometido a periodos de almacenamiento de hasta 7 días, mostraron que hay una pérdida de peso a medida que aumentaron los días de almacenamiento, llegando a perder hasta el $2 \%$ en peso con respecto al día 0 .

\section{Actividad de agua $\left(A_{w}\right)$}

Los resultados de $A_{w}$ son mostrados en la Tabla 2. El aumento de los tratamientos térmicos en $\mathrm{HA}$ utilizada al 10 $\%$ muestra una ligera tendencia a aumentar la $A_{w}$ siendo más evidente cuando se utilizó la harina tratada a $130^{\circ} \mathrm{C}$. En cambio, al incrementar el nivel de sustitución al $20 \%$ de HA no se observaron diferencias significativas entre ellas, además de que los valores obtenidos fueron menores al obtenido en HT (0.958).

La actividad de agua es la cantidad de agua libre en el alimento, es decir, la cantidad de agua disponible para reaccionar químicamente con otras sustancias y causar crecimiento microbiano. Este parámetro es muy importante en el campo de la conservación de alimentos porque es in- dicador microbiano de los alimentos y la tasa de deterioro, puede ser elemento importante para predecir el tipo de microorganismo que se desarrollará en el alimento. También es indicador de propiedades físicas, como textura, color, sabor, consistencia y aroma. En el pan, los valores de $A_{w}$ que podrían considerarse adecuados varían de 0.90 a 0.95 ; sin embargo, para este caso particular, los valores obtenidos en nuestra investigación fueron superiores a 0.95 , siendo esto más evidente cuando se utilizó $\mathrm{HA}$ al $10 \%$ y se trató a $130^{\circ} \mathrm{C}$ en la sustitución de HT para panificación, alcanzando valores superiores a 0.96. Estudios realizados por Verdú et al. (2015) al sustituir HT con harina de chía en porcentajes de 5, 10 y 15 $\%$ observaron valores muy semejantes al día 0 de la medición $\left(0.965 \mathrm{~A}_{\mathrm{w}}\right)$ mientras que en HT el mismo día de la medición obtuvieron valores de casi 0.98 de $A_{w}$.

\section{Análisis de perfil de textura (TPA)}

Los resultados relacionados con el perfil de textura de los panes elaborados con HT y HT sustituida al 10 y $20 \%$ con HA tratada térmicamente a 80,100 y $130^{\circ} \mathrm{C}$, analizados a los 0,2 y 7 días de su elaboración son mostrados en las Figuras 2,3 y 4 . La Figura 2 muestra los resultados al día 0. En cuanto a la firmeza, se observó como todas las mezclas analizadas

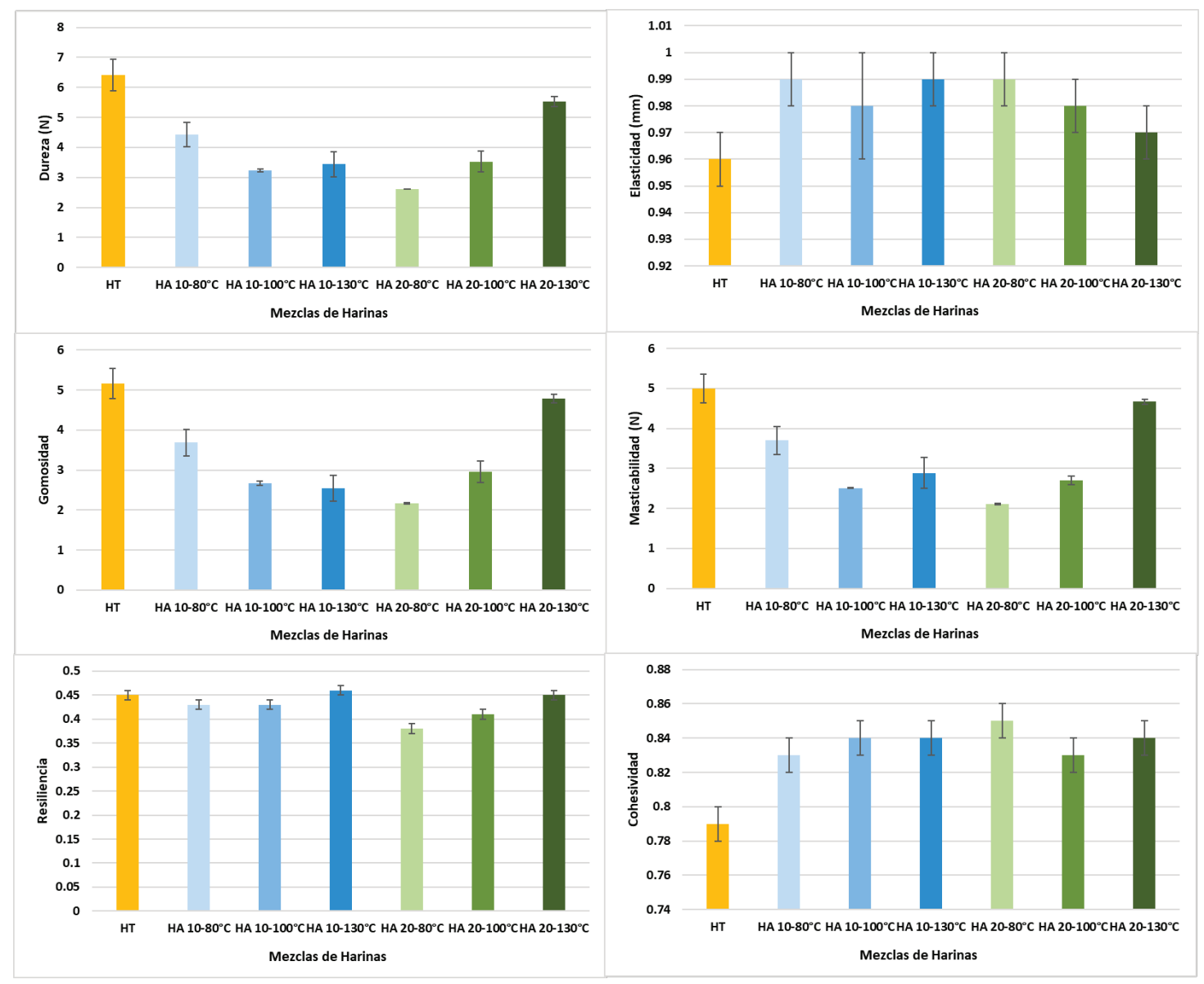

Figura 2. Análisis del perfil de textura del pan elaborado al día 0 con harina de trigo (HT) y del elaborado con la mezcla de HT con harina de avena (HA) al 10 y $20 \%$ tratada térmicamente a 80,100 y $130^{\circ} \mathrm{C}$.

Figure 2. Analysis of the texture profile of bread made on day 0 with wheat flour (HT) and that made with HT substituted with oat flour (HA) at 10 and $20 \%$, heat-treated at 80,100 and $130^{\circ} \mathrm{C}$. 
presentaron valores con diferencias significativas respecto al obtenido de HT (6.41 N). Además de lo anterior, todos los valores obtenidos resultaron ser más bajos que el de HT, de ellos destaca la mezcla HT sustituida con $\mathrm{HA}$ al $20 \%$ tratada a $80^{\circ} \mathrm{C}$, el valor alcanzado por este pan fue de $2.61 \mathrm{~N}$, un producto mucho más suave que el obtenido de HT. En cuanto a elasticidad podemos decir que no se observaron cambios importantes, valores entre 0.96 y 0.99 fueron obtenidos sin mostrar diferencias significativas entre ellos. En lo que respecta a gomosidad y masticabilidad, se observó un comportamiento similar, disminución de este parámetro a medida que aumentó el tratamiento térmico cuando se utilizó $\mathrm{HA}$ al $10 \%$, caso contrario sucedió cuando se utilizó $\mathrm{HA}$ al $20 \%$, donde hubo un incremento de gomosidad y masticabilidad a medida que aumentó el tratamiento térmico, obteniendo los valores más altos al utilizar HA al $30 \%$ (4.79 y 4.67), respectivamente. Otro parámetro analizado fue resiliencia, los valores más altos se obtuvieron cuando se analizó HT (0.45) y HT-HA al 10 y $20 \%$ tratada a $130{ }^{\circ} \mathrm{C}(0.46$ y 0.45$)$ respectivamente, sin mostrar diferencias significativas con HT. Por último, el parámetro de cohesividad se vio afectado con el nivel de sustitución de HA y el tratamiento térmico ya que se observaron diferencias significativas con HT (0.79) cuando estos fueron analizados. El pan elaborado con harina de avena mostró buenas propiedades de retención de humedad que pueden mantener el pan más suave durante períodos prolongados de tiempo (McKechnie, 1983). Tal vez esta característica tuvo una influencia importante en la firmeza del pan que se obtuvo en este estudio, ya que todas las mezclas utilizadas en el proceso de panificación resultaron en la obtención de panes más suaves que el de HT, al día 0 de almacenamiento.

La Figura 3 muestra los resultados de los parámetros analizados anteriormente, solo que al día 2 de almacenamiento. Los panes elaborados con las mezclas HT-HA al 10 $\%$ (tratada a $130{ }^{\circ} \mathrm{C}$ ), $\mathrm{HT}-\mathrm{HA}$ al $20 \%$ (tratada a $80^{\circ} \mathrm{C}$ ) y $\mathrm{HT}-\mathrm{HA}$ al $20 \%$ (tratada a $100{ }^{\circ} \mathrm{C}$ ) fueron los más suaves, incluso el obtenido de HT (17.99 N). Al analizar la elasticidad de los panes obtenidos de las diferentes mezclas de HT-HA, no se observaron diferencias significativas con respecto al obtenido de HT (0.94). Al igual que al día 0 de almacenamiento, los parámetros de gomosidad y masticabilidad mostraron un comportamiento muy similar. Cuando se utilizó HA al $10 \%$, la tendencia fue de disminuir a medida que aumentó el tratamiento térmico, observando los valores más bajos cuando se usó HA tratada a $130^{\circ} \mathrm{C}(5.79)$, en cambio cuando la sustitución de HA incrementó al $20 \%$, la tendencia fue de aumentar.

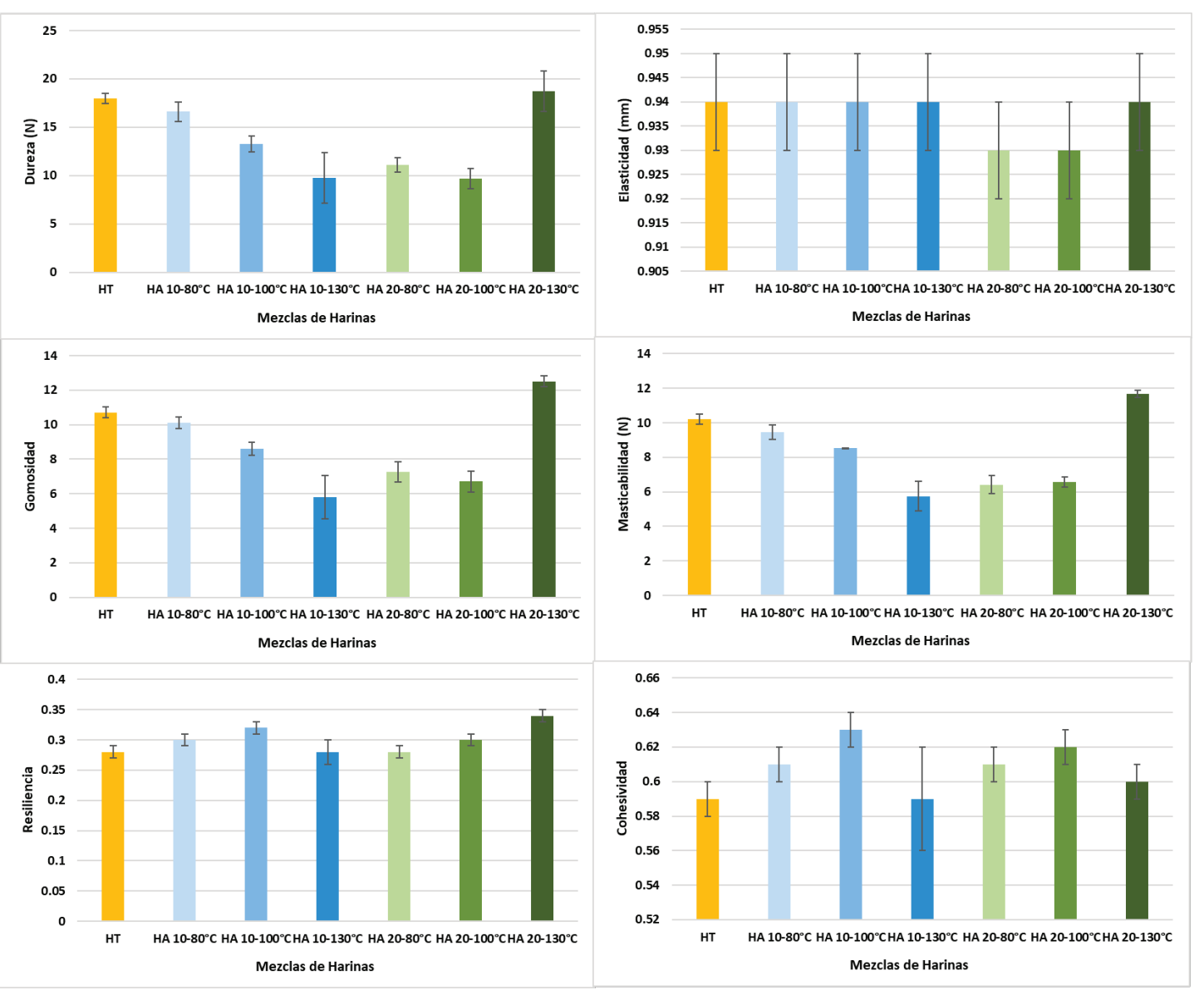

Figura 3. Análisis del perfil de textura del pan elaborado al día 2 con harina de trigo (HT) y del elaborado con la mezcla de HT con harina de avena (HA) al 10 y $20 \%$ tratada térmicamente a 80,100 y $130^{\circ} \mathrm{C}$.

Figure 3. Analysis of the texture profile of bread made on day 2 with wheat flour $(\mathrm{HT})$ and that made with $\mathrm{HT}$ substituted with oat flour (HA) at 10 and $20 \%$, heat-treated at 80,100 and $130{ }^{\circ} \mathrm{C}$. 

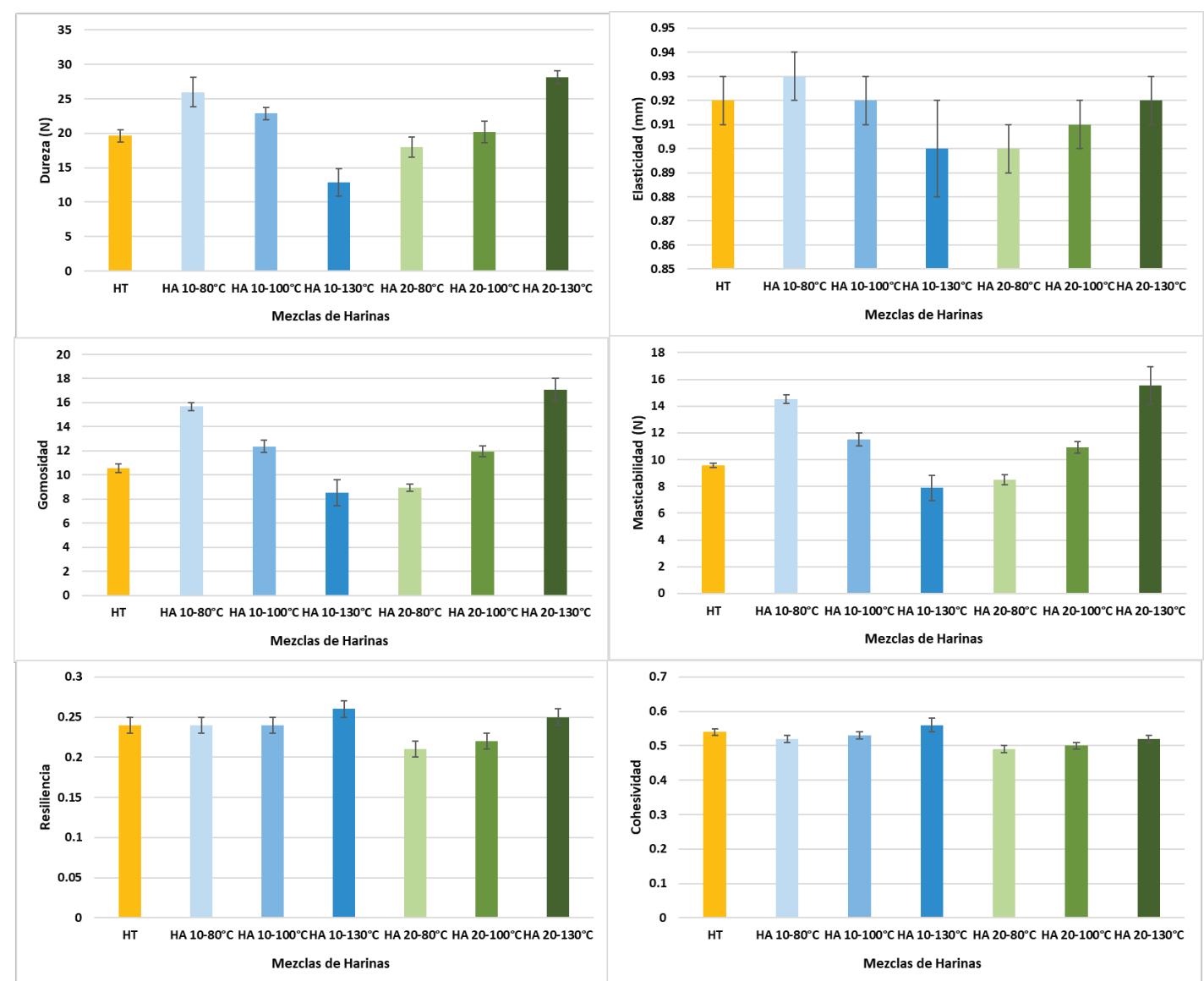

Figura 4. Análisis del perfil de textura del pan elaborado al día 7 con harina de trigo (HT) y del elaborado con la mezcla de HT con harina de avena (HA) al 10 y $20 \%$ tratada térmicamente a 80,100 y $130^{\circ} \mathrm{C}$.

Figure 4. Analysis of the texture profile of bread made on day 7 with wheat flour $(\mathrm{HT})$ and that made with $\mathrm{HT}$ substituted with oat flour (HA) at 10 and $20 \%$, heat-treated at 80,100 and $130^{\circ} \mathrm{C}$.

Este comportamiento también se observó cuando se analizó masticabilidad. En lo que respecta a resiliencia, fueron pocos los cambios observados, valores de 0.28 a 0.34 se obtuvieron en el análisis de este parámetro. Finalmente, en relación a cohesividad, al igual que elasticidad no se observaron diferencias significativas, observando valores de 0.59 a 0.63 .

Los resultados del perfil de textura obtenidos al día 7 de almacenamiento son mostrados en la Figura 4. El parámetro de firmeza mostró una tendencia a disminuir al incrementar el tratamiento térmico cuando se utilizó $\mathrm{HA}$ al $10 \%$, caso contrario fue cuando se utilizó HA al $20 \%$. Los incrementos más notables fueron observados en el pan elaborado de $\mathrm{HT}-\mathrm{HA}$ al $20 \%$ tratada a $130^{\circ} \mathrm{C}(28.19 \mathrm{~N})$. El pan de mayor suavidad se obtuvo cuando se utilizó HA al $10 \%$ $(12.86 \mathrm{~N})$ también tratada a $130{ }^{\circ} \mathrm{C}$, incluso mejoró el valor de firmeza observado en el pan elaborado de HT (19.63 N). También se analizó la elasticidad y se observó que no hubo diferencias significativas en los panes obtenidos con las diferentes mezclas de HT-HA, valores que resultaron muy similares a los obtenidos en el día 2 de almacenamiento. Los parámetros de gomosidad y masticabilidad mostraron un comportamiento muy similar al día 2 de almacenamiento, al igual que se observó en elasticidad, aunque también valores más altos en estos parámetros fueron obtenidos cuando se utilizó HA al $20 \%$ (17.09 y 15.54, respectivamente) tratada térmicamente a $130^{\circ} \mathrm{C}$.

Finalmente, parámetros como resiliencia y cohesividad tienden a disminuir a medida que aumentaron los días de almacenamiento. Rangos de entre 0.21 a 0.25 fueron observados para resiliencia, mientras que para cohesividad fueron de 0.49 a 0.54 . Las mediciones de compresibilidad como lo son la medición del perfil de textura o TPA son un indicativo importante de cómo los consumidores perciben diferencias en el envejecimiento del pan. El envejecimiento del pan se refiere a los cambios indeseables que se presentan (además de la contaminación microbiana) entre el tiempo que pasa desde que el pan se elabora hasta que se consume. Comprendiendo los diferentes aspectos del envejecimiento y los factores que lo afectan, puede ayudar al productor a tomar las mejores decisiones en cuanto a las fórmulas, ingredientes y empaques a utilizar.

Estudios realizados por Martin et al. (1991) mostraron que la firmeza del pan es el resultado de la formación de puentes de hidrógeno entre los gránulos de almidón gelatinizado y la red de gluten en el pan, enlazándose juntos a la red de proteína continua y los gránulos remanentes dis- 
continuos. También propusieron que durante el horneado se forman enlaces cruzados entre el almidón y el gluten, y durante el almacenamiento el número de interacciones como su fuerza se incrementan. Otros investigadores como Gerrard et al. (2001) concluyeron que la firmeza de la miga no está significativamente correlacionada con el tipo de proteínas de la harina o con su concentración. Londono et al. (2015) estudiaron el papel de $\beta$-glucanos de avena en sistemas de masas, concluyeron que masas hechas de harinas bajas en $\beta$-glucanos $(<2 \%)$ mostraban una mayor capacidad de retención de gas. El impacto de $\beta$-glucanos en las propiedades de los sistemas de masa de avena está regulado por la concentración y viscosidad. Los $\beta$-glucanos son componentes claves que determinan la reología de masas donde está involucrada harina de avena, además de la funcionalidad tecnológica en los sistemas de masa (Hüttner et al., 2010). Lo anterior puede ser una consideración muy importante que influye en las propiedades relacionadas con el perfil de textura del pan obtenido en este estudio.

\section{CONCLUSIONES}

Los parámetros de viscosidad se vieron afectados por el nivel de sustitución de la harina de avena y por el tratamiento térmico al que fue sometida. Estos efectos fueron más evidentes cuando la harina de avena se trató térmicamente a $130^{\circ} \mathrm{C}$. Todos los tratamientos térmicos aplicados en harina de avena utilizada en la sustitución de harina de trigo favorecieron el volumen específico del pan, esto fue para los dos niveles de sustitución (10 o $20 \%$ ), sin embargo, cuando se utilizó harina de avena al $20 \%$ tratada a $100{ }^{\circ} \mathrm{C}$ fue que se observó el mayor volumen, incluso mejor al obtenido del pan elaborado solo de harina de trigo. El uso del $20 \%$ de la harina de avena tratada a $80^{\circ} \mathrm{C}$ en la sustitución de la harina de trigo mejoró el valor de la firmeza, disminuyendo más que cualquiera de los tratamientos medidos en el día 0 , incluso al obtenido al medir el pan elaborado solo con harina de trigo.

La sustitución del $10 \%$ con harina de avena tratada a $130^{\circ} \mathrm{C}$ favoreció la elaboración de un pan más suave, al medir este parámetro a los 7 días de almacenamiento.

\section{REFERENCIAS}

AACC, 2000. Approved Methods of American Association of Cereal Chemists. 10th Ed. The Association, St. Paul, Minnesota, USA. Methods 56-11.02, 76-21.02.

Batey, I. L. 2009. Interpretation of RVA Curves. In G. B. Crosbie \& A. S. Ross (Eds.). The RVA Handbook (pp. 19-30). USA: AACC International.

Berton, B., Scher, J., Villieras, F. y Hardy, J. 2002. Measurement of hydration capacity of wheat flour: Influence of composition and physical characteristics. Powder Technology (128) 326331.

Cauvain, S. P., Hodge, G., Muir, D. M. y Dodds, N. J. 1976. Treatment of grain. U.S., A23B 4/04.

Catterall, P.F. 2001.The production of cakes from non-chlorinated cake flour. AACC, Annual meeting. Charlotte, North Carolina.

Chesterton, A. K. S., Wilson, D. I., Sadd, P. A. y Moggridge, G. D. 2015. A novel laboratory scale method for studying heat treatment of cake flour. Journal of Food Engineering (144) 36-44.

Cordero, D. L., Granados, M. C., Islas, A. R., Verdú, S., Ramírez, B. y Vásquez, F. 2020. Utilización de fibra de avena con diferente tamaño de partícula en panificación: efecto reológico y textural. Revista Mexicana de Ciencias Agrícolas, Vol. 11 (1) 161-173.

Duyvejonck, A. E., Lagrain, B., Pareyt, B., Courtin, C. M. y Delcour, J. A. 2011. Relative contribution of wheat flour constituents to solvent retention capacity profiles of European wheats. Journal of Cereal Science (53) 312-318.

Gelinas, P., Mckinnon, C. M., Rodrigue, N. y Montpetit, D. 2001. Heating conditions and bread-making potential of substandard flour. Journal of Food Science (66) 627-632.

Gerrard, J. A., Abbot, R.C., Newberry, M. P. Gilpin, M. J., Ross, M. y Fayle, S. E. 2001. The effect of nongluten proteins on the staling of bread. Starch 53(6): 278-280.

Hanamoto, M. y Bean, M. 1979. Process for improving baking properties of unbleached cake flours. US, A21D6/00.

Haynes L. C., Bettge A. D. y Slade, L. 2009. Soft wheat and flour products methods review: solvent retention capacity equation correction. AACC International Report 54, 174-175.

Hüttner, E. K., Dal Bello, F. y Arendt, E. K. 2010. Rheological properties and bread making performance of commercial wholegrain oat flours. Journal of Cereal Science (52) 65-71.

Jiranuntakul, W., Puttanlek, Ch., Rungsardthong, V. y Punchaarnon, S. 2011. Microstructural and physicochemical properties of heat-moisture treated waxy and normal starches. Journal of Food Engineering (104) 246-258.

Ktenioudaki, A., O'shea, N. y Gallagher, E. 2013. Rheological properties of wheat dough substituted with functional byproducts of food processing: Brewer's spent grain and apple pomace. Journal of Food Engineering (116) 362-368.

Lei, F., Ji Chung, T., Cai Ling, S. y Chun, L. 2008. RVA and farinograph properties study on blends of resistant starch and wheat flour. Agricultural Science in China 7 (7) 812-822.

Londono, Diana M., Gilissen, Luud J.W.J., Visser, Richard G.F., Smulders, Marinus J.M. y Hamer, Rob J. 2015. Understanding the role of oat $\beta$-glucan in oat-based dough systems. Journal of Cereal Science (62) 1-7.

McKechnie, R., 1983. Oat products in bakery foods. Cereal Foods World (28) 635-637.

Marston, K., Khouryieh, H. y Aramouni, F. 2016. Effect of heat treatment of sorghum flour on the functional properties of gluten-free bread and cake. LWT-Food Science and Technology (65) 637-644.

Martin, M., Zeleznak, K. y Hoseney, R. 1991. A mechanism of bread firming. I. Role of starch swelling. Cereal Chemistry 68(1): 498-503.

Miñarro, B., Albanell, E., Aguilar, N., Guamis, B. y Capellas, M. 2012. Effect of legume flours on baking characteristics of gluten-free bread. Journal of Cereal Science (56) 476-481.

Nakamura, C., Yoshiki, K. y Seguchi, M. 2008. Increased volume of Kasutera cake by dry heating of wheat flour. Food Science and Technology Research (14) 431-436.

Neill, G., Al-Muhtaseb, A. H. y Magee, T. R. A. 2012. Optimisation of time/temperature treatment, for heat treated soft wheat flour. Journal of Food Engineering (113) 422-426.

Ozawa, M., Kato, Y. y Seguchi, M. 2009. Investigation of dryheated hard and soft wheat flour. Starch-Stärke, 61(7), 398406. 
Pauly, A., Pareyt, B., Fierens, E. y Delcour, J. A. 2013. Whet (Triticum aestivum L. and T. turgidum L. ssp. durum) wheat kernel hardness: II. Implications there. Compr. Rev. Food Sci. Saf. 12 (4) 427-438.

Prakash, M. y Haridas, R. P. 1999. Effect of steaming on the rheological characteristics of wheat flour dough. European Food Research and Technology 209(2), 122-125.

Protonotariou, S., Drakos, A., Evangeliou, V., Ritzoulis, C. y Mandala, I. 2014. Sieving fractionation and jet mill micronization affect the functional properties of wheat flour. Journal of Food Engineering (134) 24-29.
Pyler, E. J. 1988. Cake baking technology. Baking science and technology (979-1027). Kansas City: Sosland Publishing.

Russo, J. V. y Doe, C. A. 1970. Heat treatment of flour as an alternative to chlorination. Journal of Food Technology (5) 363-374.

Serna-Saldívar, S. O. 1996. Química, almacenamiento e industrialización de los cereales. AGT, Editores. S.A.

Verdú, S., Vásquez, F., Ivorra, E., Sánchez, A. J., Barat, J. M. y Grau, R. 2015. Physicochemical effects of chia (Salvia hispánica) seed flour on each wheat bread-making process phase and product storage. Journal of Cereal Science (65) 67-73. 